\title{
Cooperating Coscheduling in a Non-dedicated Cluster $^{\star}$
}

\author{
Francesc Giné ${ }^{1}$, Francesc Solsona ${ }^{1}$, Porfidio Hernández ${ }^{2}$, and Emilio Luque ${ }^{2}$ \\ 1 Departamento de Informática e Ingeniería Industrial, Universitat de Lleida, Spain. \\ $\{$ sisco,francesc $\} @ e u p . u d l . e s$ \\ 2 Departamento de Informática, Universitat Autònoma de Barcelona, Spain. \\ $\{$ p.hernandez, e.luque $\} @ c c . u a b . e s$
}

\begin{abstract}
Our research is focussed on keeping both local and parallel jobs together in a time-sharing NOW. In such systems, new scheduling approaches are needed to allocate the underloaded resources among parallel jobs. In this paper, a distributed system, named Cooperating CoScheduling, is presented to assign CPU and memory resources efficiently by applying resource balancing and dynamic coscheduling between parallel jobs. This is shown experimentally in a PVM-Linux NOW.
\end{abstract}

\section{Introduction}

Time-slicing scheduling techniques [14] exploit the unused resources of a Network Of Workstations (NOW), by running both parallel and local jobs together. In this framework, the coscheduling of parallel jobs becomes a critical issue. Some studies [6]5] have shown the good performance of dynamic coscheduling techniques when many local users compete with a single parallel job. Dynamic coscheduling deals with minimizing the communication waiting time of parallel processes. With this aim, when parallel tasks are dynamically coscheduled, the arrival of a message will cause the dispatch of the target task. Unfortunately, dynamic coscheduling performance can slow down drastically when the number of parallel jobs competing against each other (MultiProgramming Level (MPL)) is increased. This is due to the fact that if the resources are not well-balanced between the competing parallel tasks, the completion of each process of a parallel job will require a different amount of time, and the entire job must wait for the slowest process before it can synchronize.

A new technique, called Cooperating CoScheduling (CCS), is presented in this paper. CCS improves dynamic coscheduling techniques by balancing the CPU and memory resources between parallel jobs throughout the NOW. Each node manages its resources efficiently by combining local - basically communication events, local user logging and computational capabilities-, and foreign runtime information (i.e. the local user logging onto a remote node) provided by their cooperating nodes. CCS is implemented in a PVM-Linux NOW.

\footnotetext{
* This work was supported by the MCyT under contract TIC 2001-2592 and partially supported by the Generalitat de Catalunya - Grup de Recerca Consolidat 2001SGR00218.
} 


\section{CCS: Cooperating CoScheduling}

Our framework is a non-dedicated NOW, where all nodes are under the control of the CCS scheme. In each node, a time-sharing scheduler is assumed with task preemption based on the ranking of tasks according to their priority. The scheduler works by dividing the CPU time into epochs. In every epoch, each task is assigned a specific time slice and priority. In order to give preference to $\mathrm{I} / \mathrm{O}-$ bound processes, the priority of each task is inversely proportional to the CPU used during the last epoch. A Memory Management Unit is assumed with demand-paging virtual memory. A SPMD model is assumed for parallel jobs. We assume that each node applies a dynamic coscheduling technique [5]. This means that every local scheduler increases the receiving task priority according to the number of packets in its reception queue, even causing CPU preemption of the task being executed inside.

The need to preserve local job performance limits the resources assigned to parallel tasks. This may lead to non-desirable situations (see fig. 11(a)). A parallel job $\left(J_{1}\right)$ is executed on node $_{1}$ and node $_{2}$ with $25 \%$ of resources (CPU and Memory), while the local user has the remaining $75 \%$ available. Simultaneously, another parallel job $\left(J_{2}\right)$ is executed with full resources on node $_{4}$ and node . Meanwhile, node $_{3}$ assigns half of the resources to each parallel job following a fairness policy. This uneven taxing of resources creates a situation where some processes of the parallel program run slower than others. However, if node $_{3}$ had knowledge about the status of $J_{1}$ and $J_{2}$ jobs in the remaining nodes (cooperating nodes), it could take better decisions by giving $J_{2}$ more resources than $J_{1}$. Thus, the situation shown in fig. I (b) would be achieved. In this case, $J_{1}$ has the same amount of resources in each node, while $J_{2}$ has more resources available than in the previous case. This way, the NOW resources are better balanced.

This example illustrates the three main aims of the CCS algorithm, namely:

a) the performance of local jobs; b) the uniform allocation of the CPU and memory resources assigned to each parallel job throughout the cluster; and c) the coscheduling of communicating parallel jobs.

\subsection{Cooperating CoScheduling Mechanism}

This section explains how CCS achieves the objectives set out above.

Job interaction. We use priority as a mechanism to limit the interference of parallel jobs in the resources (CPU and memory) needed by local tasks. In the CPU, when node $_{i}$ detects the presence of a local user, it will assign a time slice to parallel and locals tasks equal to $D E F_{-} Q U A N T U M_{i} * L$ and $D E F_{-} Q U A N T U M_{i} *(1-L)$, respectively. $D E F_{-} Q U A N T U M_{i}$ is the base time slice of node $_{i}$ and $L$ is the minimum percentage of resources assigned to parallel jobs. The minimum term is related to the fact that if parallel tasks require a bigger percentatge of CPU than $L$, then they will be able to use the portion allocated to local tasks, whenever local tasks are not using this. It is assumed that $L$ has the same value across the cluster. In order to quantify the heterogeneous nature of a NOW, we have used the Power Weight [1] $\left(W_{i}=B O G O_{i} / B O G O_{\max }\right)$, 


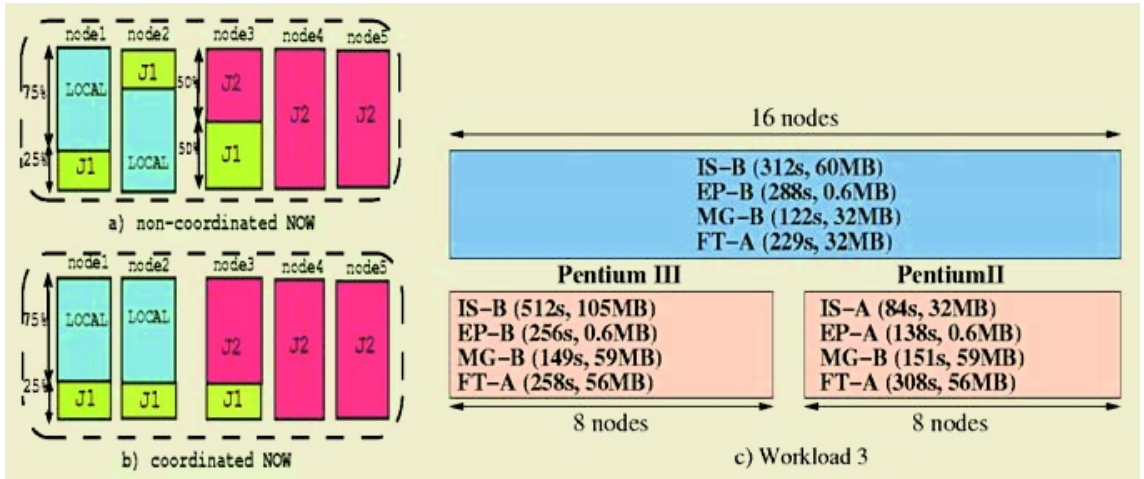

Fig. 1. (a and b) Example of applying CCS. (c) Workload 3.

where $1<=i<=n$ (number of nodes) and $B O G O_{i / \max }$ is a measurement of the computational capabilities of the $i t h$ and fastest node, respectively. Note that this measurement is provided by some o.s (p.e. Linux) in the boot period. Thus, the base time slice of each node is set relative to its Power Weight $\left(D E F_{-} Q U A N T U M_{i}=D E F_{-} Q U A N T U M / W_{i}\right)$, where $D E F_{-} Q U A N T U M$ is the base time slice of the local o.s. (p.e. Linux=210ms).

The memory $\left(M_{i}\right)$ is divided into two pools: one for the local tasks $\left(M_{i}^{L}\right)$ and the other for parallel jobs $\left(M^{D}=\min _{i=1}^{n}\left(M_{i}\right) * L\right)$. Note that the same minimum memory size $\left(M^{D}\right)$, will be guaranteed to parallel tasks in each node. As in the CPU case, if parallel tasks require a bigger memory size than $M^{D}$ then they will be able to use the portion allocated to local tasks, whenever local tasks are not using this. However, in the nodes where swapping is activated and the memory requirements of the parallel tasks exceed their assigned part $\left(M^{D}\right)$, the parallel task with most pages mapped in memory (swapping_task) will be stopped. Consequently, the resident size of the remaining tasks will be increased and the swapping activity will also be reduced 2 .

Cooperating Scheme. In order to balance resources throughout the cluster, the cooperating nodes should interchange status information. The key question is which information should be interchanged. An excess of information could slow down the performance of the system, increasing its complexity and limiting its scalability. For this reason, only the following four events are notified:

1. $L O C A L$ : when there is local user activity in a node, it sends the identifier of the parallel jobs executing in such node to all its cooperating nodes.

2. NO_LOCAL: if the local activity finishes, the node sends a notification message to its cooperating nodes.

3. STOP: when swapping_task is stopped, the node sends the identifier of the penalized job to its cooperating nodes.

4. RESUME: when the swapping_task restarts, the node informs its cooperating nodes of this event. 
When a cooperating node receives one of the above events, it will reassign the resources according to the following rules. (1) The scheduler with more than one parallel tasks under its management will assign a quantum equal to $\left(D E F_{-} Q U A N T U M_{i} * L\right)$ or zero to those tasks notified by LOCAL or STOP events, respectively. (2) In order to ensure that a parallel job is not slowed down by any local user across the cluster, a node will only reassign more resources to a task notified by a $N O_{-} L O C A L$ event when the number of $L O C A L$ received events coincides with the $N O_{-} L O C A L$ received events. (3) If a node receives a STOP notification for a parallel task, this task will be chosen as swapping_task, whenever the memory is exhausted. (4) If a node with overloaded memory receives the $S T O P$ event for a task different than the swapping_task, the kernel will stop the notified task and the swapping_task will be resumed. (5) If a node with overloaded memory receives a RESUME event associated with the swapping_task, the kernel will restart the notified task and a new swapping_task will be chosen. Applying these rules, we show experimentally (see extended version [3]) that the cluster reaches a stable state.

\section{Experimentation}

CCS was implemented and tested in a PVM (v.3.4) - Linux (v.2.2.15) cluster. A complete description of the CCS implementation can be found in [3]. Our cluster was composed of eight Pentium II and eight Pentium III with 128 and $256 \mathrm{MB}$ of memory and a Power Weight $\left(W_{i}\right)$ of 0.43 and 1, respectively. All of them were connected through a Fast Ethernet network.

The performance of the parallel jobs was evaluated by running four PVM benchmarks from the NAS suite with class A and B: IS and FT, MG and EP. Three workloads (Wrk1, Wrk2 and Wrk3) with different job sizes and MultiProgramming Level (MPL) were defined. Wrk1 is characterized by a MPL equal to 1 , while $W r k 2$ and $W r k 3$ are 2. Wrk1 continuously executes a benchmark of size 16 chosen randomly according to a discrete uniform distribution. Wrk2 continuously executes two size 16 jobs with the constraint that parallel tasks fit in their assigned memory portion $\left(M^{D}\right)$. Wrk3 executes one size 16 job together with two size 8 jobs (see fig. 1(c)) without any memory constraint. Additionally, fig. 1(c) shows the execution time and memory requirements of every benchmark on a dedicated system. Each workload was executed for 4 hours.

The local workload was carried out by running one synthetic benchmark, called local. This allowed the CPU load, memory requirements and network traffic used by the local user to be fixed. According to the monitoring of real local users [3], we defined two different local user profiles: a user with high CPU requirements, denoted as $C P U$, and a user with a high requirements for interactivity, denoted as Xwin. These parameters were set at $(0.65,35 \mathrm{MB} 1$ and $0 \mathrm{~KB} / \mathrm{s})$ and $\left(0.20,84 M B^{1}\right.$ and $\left.3 \mathrm{~KB} / \mathrm{s}\right)$ for $C P U$ and $X$ win users, respectively. In order to simulate the high variability of local user behavior, the local execution time was modeled by a two stage hyper-exponential distribution with means,

\footnotetext{
${ }^{1}$ Memory requirements do not include kernel memory.
} 

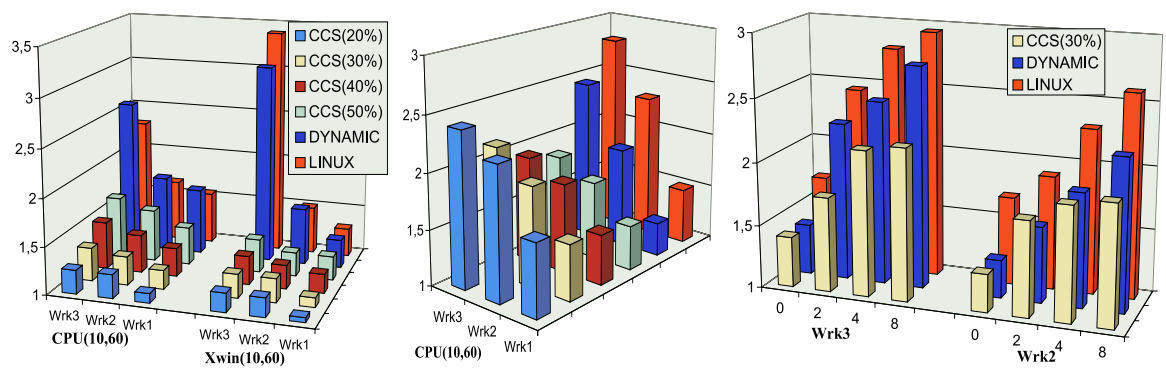

Fig. 2. (left) Slowdown of local and (center) parallel tasks with four local users. (right) Slowdown of parallel tasks varying the number of local CPU users.

by default, of $10 \mathrm{~min}$ and $60 \mathrm{~min}$ and a weight of 0.6 and 0.4 for each stage. At the end of its execution, the local benchmark returns the system call latency, memory and network bandwidth and wall-clock execution time.

Three environments were evaluated: the plain LINUX, the DYNAMIC and the CCS schedulers. Note that the DYNAMIC scheduler applies exclusively a dynamic coscheduling technique without any restrictions of the resources used by parallel jobs and any exchanging of information between cooperating nodes.

The performance of the parallel and local jobs was validated by means of the Slowdown metric (which is the average of 5 different executions). The next section shows a summary of the extended version of the experimentation [3].

\subsection{Experimental Results}

Fig. 2 (left) shows the slowdown of local according to the percentage of resources assigned to parallel tasks $(L)$. $L$ was varied between $20 \%$ to $50 \%$. This trial was repeated twice: once with only CPU users and again with Xwin users. We can see that LINUX and PREDICTIVE gave the worst performance for local tasks. The peak obtained with the $W r k 3$ is because the Wrk3 memory requirements exceed the main memory size of some nodes with local activity and as a consequence, swapping is performed without guaranteeing any minimal memory size to local tasks. With the exception of the Wrk3 case, it is worth pointing out the low intrusion for the Xwin users. This is due to the priority given by Linux to I/O processes. The impact of CCS rises slightly when the MPL is increased to 2 (Wrk2 and Wrk3), although this is always limited to below values of 1.25 and 1.6 for Xwin and CPU users respectively, even in the worst case for local tasks $(L=50 \%)$. For the rest of the trials, the local workload is exclusively made up of CPU local users (the most unfavorable case for parallel performance).

Fig. 2 (center) shows the slowdown in the performance of parallel jobs under the same conditions explained above. In general, LINUX obtains the worst results due mainly to the absence of coscheduling between communicating tasks. $D Y N A M I C$ obtains the best results for Wrk1 while CCS obtains the best results for Wrk3. This degradation for DYNAMIC in the Wrk3 case is due to the fact 
that high or moderate page fault frequency in one node can drop parallel job performance drastically, vastly exceeding in this way the coscheduling benefits. In the case of Wrk2, CCS obtains better results than DYNAMIC for $L \geq 30$. This is due to the fact that the resources are better balanced under CCS and thus, all PVM jobs can progress across the NOW in a coordinated way. Fig. 2 (right) shows the slowdown on Wrk2 and Wrk3 when the number of local users was varied between 0 and 8. For the Wrk2 case, $C C S$ maintains a nearly constant slowdown in a non-dedicated environment, while DYNAMIC performance is slightly degraded when local users are increased. This is due to the absence of a mechanism for balancing resources across the NOW. Regarding the Wrk3 case, $C C S$ obtains the best results with this workload because $C C S$ can distribute NOW resources better between parallel jobs of different sizes.

\section{Conclusions and Future Work}

In this paper, a new technique, named Cooperating CoScheduling (CCS), is presented. The main aim of $C C S$ is to exploit the unused computing capacity of a non-dedicated cluster without disturbing local jobs excessively. This is achieved by means of combining balancing of resources and coscheduling between parallel jobs. In doing so, each CCS node assigns its resources dynamically, based on a combination of local and foreign runtime information provided by its own o.s. and its cooperating nodes, respectively. Thus, local decisions are taken in a coordinated way across the NOW. The performance of $C C S$ has been tested in a PVM-Linux cluster and compared with other coscheduling policies. The results obtained demonstrated its good behavior, substantially reducing the response time of local tasks and improving, in some cases, the slowdown of parallel tasks with respect to other coscheduling policies.

Our next work is directed towards extending $C C S$ to be applied together with a space-slicing technique. Our aim is to execute multiple parallel programs in every cluster partition by means of applying the CCS technique in each one.

\section{References}

1. X. Du and X. Zhang. Coordinating parallel processes on networks of workstations. Journal of Parallel and Distributed Computing, 46(2):125-135, 1997.

2. F. Giné, F. Solsona, P. Hernández, and E. Luque. Minimizing paging tradeoffs applying coscheduling techniques in a linux cluster. LNCS, 2565:593-607, 2002.

3. F. Giné, F. Solsona, P. Hernández, and E. Luque. Cooperating coscheduling in a nondedicated cluster. extended version. Technical Report DIEI-03-RT-1, Universitat de Lleida, 2003.

4. K.D. Ryu and J.K. Hollingsworth. Linger longer: Fine-grain cycle stealing for networks of workstations. In Proceedings Supercomputing'99 Conference, 1999.

5. P.G. Sobalvarro, S. Pakin, W.E. Weihl, and A.A. Chien. Dynamic coscheduling on workstation clusters. LNCS, 1459:231-256, 1998.

6. F. Solsona, F. Giné, P. Hernández, and E. Luque. Predictive coscheduling implementation in a non-dedicated linux cluster. LNCS, 2150:732-741, 2001. 